\title{
Absence of Escherichia coli, Listeria monocytogenes, and Klebsiella pneumoniae antigens within inflammatory bowel disease tissues
}

\author{
R S Walmsley, A Anthony, R Sim, R E Pounder, A J Wakefield
}

\begin{abstract}
Background-Escherichia coli, listeria, and streptococcal antigens have been found in Crohn's disease tissues. Antibodies to Klebsiella pneumoniae have been found in patients with inflammatory bowel disease and ankylosing spondylitis. The presence of these bacterial antigens in Crohn's granulomas would be of aetiological interest, while their presence in ulcers alone would be more likely to indicate secondary infection.
\end{abstract}

Aim-To investigate inflammatory bowel disease tissues for the presence of these bacteria.

Methods-Formalin fixed, paraffin processed sections from 53 patients (19 ulcerative colitis, 23 Crohn's disease; 11 normal tissues from cancer resections) were studied by immunohistochemistry. Control tissue consisted of normal human small bowel injected submucosally with either $E$ coli, Listeria monocytogenes, Proteus mirabilis, or Klebsiella pneumoniae serotypes $\mathrm{K} 2,3,17,21,26,36$, and 50 , and colonic biopsies from a child with $E$ coli 0114 infection. Tissues were stained by Gram-Twort, and with specific antibodies for $E$ coli (Dako B357), L monocytogenes (Difco 2302-50), and $K$ pneumoniae (Biogenesis 5580-5208) using an immunoperoxidase technique.

Results-Positive staining for $E$ coli was observed on the luminal surface epithelium and in ulcers in 35\% of Crohn's disease patients, $26 \%$ of ulcerative colitis patients, and no normal controls. Superficial staining for $L$ monocytogenes was observed in one case of ulcerative colitis only. Staining for $K$ pneumoniae was observed in one case of ulcerative colitis and one of Crohn's disease. No granulomas, giant cells, or germinal centres stained positively for any of the three bacterial antigens.

Conclusions-These data do not support a primary role for $E$ coli, $L$ monocytogenes, and $K$ pneumoniae in inflammatory bowel disease. The presence of $E$ coli antigens in ulcers suggests secondary infection in these lesions.

(F Clin Pathol 1998;51:657-661)

Keywords: Crohn's disease; Escherichia coli; Listeria monocytogenes; Klebsiella pneumoniae

The intestinal bacterial flora may have an important contributory role in the pathology of inflammatory bowel disease. Indirect evidence for this includes the finding that diversion of the faecal stream can prevent recurrence of Crohn's disease following terminal ileal resection ${ }^{1}$; that treatment with metronidazole for three months following resection halves the relapse rate at one year ${ }^{2}$; that systemic endotoxaemia is related to both disease extent and severity in both ulcerative colitis and Crohn's disease ${ }^{3}$; and that specific cytokine knock-out mice develop colitis when their bowel becomes colonised with the normal bacterial flora but do not do so when reared in a germ-free environment. ${ }^{45}$ In the search for specific bacterial pathogens, analysis of stool cultures have suggested higher numbers of anaerobic Gram negative rods and coccoid-rods of the Eubacterium and Peptostreptococcus species in Crohn's disease patients, ${ }^{6}$ and studies of the humoral response have found antibodies not only to these species $^{7}$ but also to Escherichia coli, Yersinia enterocolitica, ${ }^{8}$ and Klebsiella pneumoniae. ${ }^{9}{ }^{10}$ Klasen et al, using immunocytochemical techniques, found evidence of complexes of peptidoglycan-polysaccharide, a component of the cell wall of Gram positive bacteria, in Crohn's disease tissues, and demonstrated a cellular immune response to these complexes. ${ }^{11}$ More recently Liu et al undertook an extensive immunohistochemical search for evidence of both viral and bacterial antigens in French patients with familial Crohn's disease. ${ }^{12}$ They found that $75 \%$ of the bowel sections stained positive for Listeria monocytogenes, $57 \%$ for $E$ coli, and $44 \%$ for streptococcal species. Positively stained macrophages and giant cells were distributed beneath ulcers, along fissures, and in abscesses, granulomas, and germinal centres. The apparent presence of these antigens in Crohn's granulomas-localising reactions to potentially causative antigens - is of particular interest.

The aim of this study was to examine tissue sections obtained from resection specimens in patients with Crohn's disease and ulcerative colitis for the bacterial antigens of Escherchia coli, Listeria monocytogenes, and Klebsiella pneumoniae using immunohistochemical techniques.

Methods

TISSUES

We examined formalin fixed and paraffin processed sections from resected bowel specimens of 53 patients. Twenty three sections were from
Accepted for publication 13 May 1998 

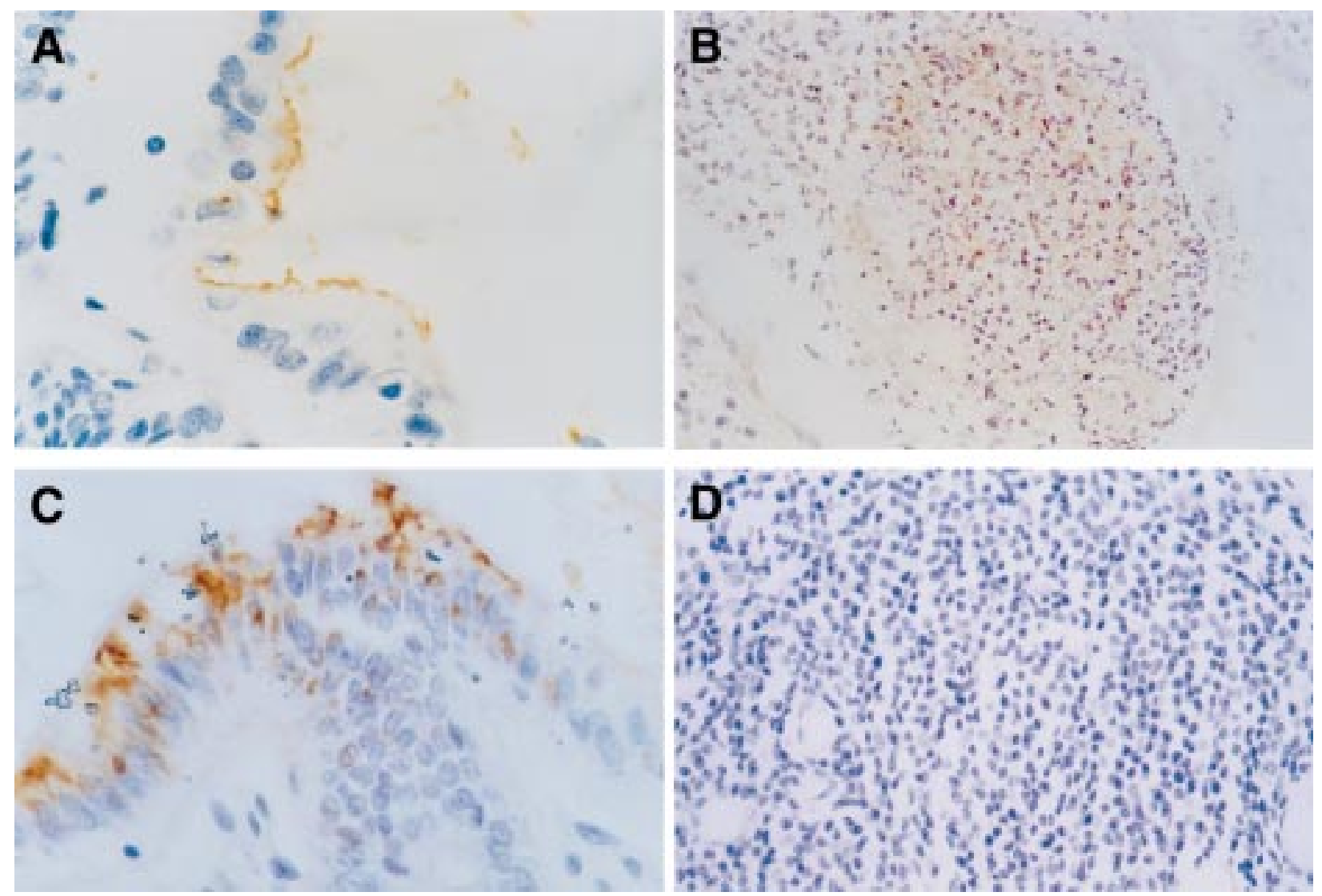

Figure 1 Immunohistochemical staining for Escherichia coli antigen by immunoperoxidase technique developing with DAB. (A) Positive control colonic biopsy from a child with $E$ coli $O 114$ infection. The organisms are closely adherent to the epithelial surface. (B) Positive staining for $E$ coli antigens in a crypt abscess of a case of Crohn's disease. (C) Positive epithelial staining in a case of ulcerative colitis. (D) A lymphoid aggregate at the base of a fissure in a case of Crohn's disease showing negative staining for $E$ coli antigen.

Crohn's disease resection specimens, in one case with the addition of the resected lymph nodes. The cases were unrelated and were chosen on the basis that they showed the characteristic changes of Crohn's disease - that is granulomas, fissuring, ulceration, lymphoid aggregates, and transmural inflammation. Nineteen cases of ulcerative colitis were similarly examined: 15 were sections from colectomy specimens and four were colonoscopic biopsies. As controls we examined 11 sections of normal colon obtained from resection specimens taken from cases of malignancy. Positive control tissue consisted of $1 \mathrm{~cm}^{2}$ sections of normal human small bowel injected submucosally with $250 \mu$ of bacterial cultures containing approximately $1 \times 10^{11} / \mathrm{ml}$ of bacteria: $E$ coli National Tissue and Cell Collection (NTCC) B35, L monocytogenes NTCC 7973, Proteus mirabilis, K pneumoniae serotypes K2, 3, $17,21,26,36$, and 50, and colonic biopsies from a child with $E$ coli 0114 infection. The $E$ coli, $L$ monocytogenes, and $P$ mirabilis were all clinical isolates obtained by the Department of Microbiology, King's College, London. $K$ pneumoniae serotypes were donated by $\mathrm{Dr} T$ Pitt, Public Health Services Laboratories, Colindale, UK.

IMMUNOHISTOCHEMISTRY TECHNIQUES

All sections underwent both routine haematoxylin and eosin staining, to confirm the diagnosis and characterise morphology, and Gram-
Twort staining to facilitate localisation of Gram negative organisms within tissues. ${ }^{13}$

An immunoperoxidase ABC-DAB technique was used for the specific staining for bacteria (Dako Duet kit, Dako, High Wycombe, UK). Sections were deparaffinised through xylene to alcohol, and endogenous peroxidase was blocked with 3\% hydrogen peroxide for 10 minutes. After washing with Tris buffered saline (TBS) for eight minutes, non-specific binding was blocked by addition of normal goat serum diluted 1 in 10 in TBS for 20 minutes. The primary antibody was then added for the appropriate time (see below) and the slides washed again in TBS for eight minutes. Secondary antibody (goat anti-rabbit/ mouse) at a dilution of 1 in 200, with normal human serum at 1 in 25 in TBS, was added for 30 minutes at room temperature. Further washing was followed by the addition of the streptavidin-biotin complex $(1+1: 200)$ for 30 minutes. A final wash with TBS was followed by development with diaminobenzidine (DAB). Counterstaining with Meyer's haematoxylin was followed by differentiation in $0.5 \%$ acid alcohol, bluing, and dehydration back to xylene.

Sections were stained for three bacteria. For $E$ coli a polyclonal antibody was used (Dako, B357) at a dilution of 1 in 6000, applied for 30 minutes at room temperature. In addition, 10 Crohn's disease sections, three normal sections, and control sections were stained over- 


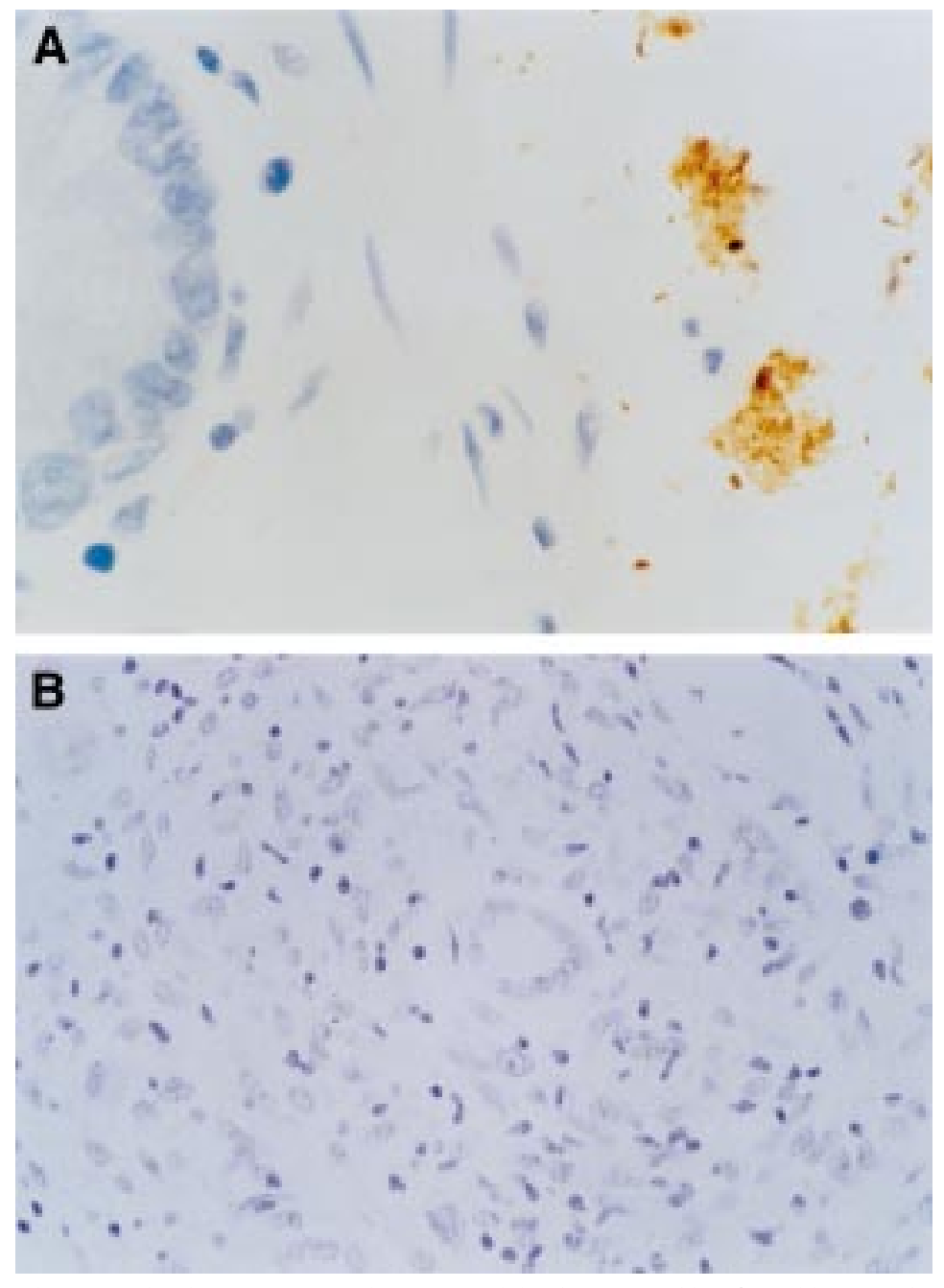

Figure 2 Listeria monocytogenes immunoreactivity demonstrated in (A) positive control section of normal human small bowel injected submucosally with L monocytogenes NCTC 7973. (B) Negative staining of a typical granuloma from a Crohn's disease case.

night at $4^{\circ} \mathrm{C}$, according to Liu et al..$^{12}$ There was no difference in sensitivity between the two methods. For $L$ monocytogenes we used a polyclonal antibody (catalogue No 2302-50, Difco, Detroit, Michigan, USA) at 1 in 4000 dilution for 30 minutes. Again, assessment of overnight staining at a dilution of 1 in 50000 at $4^{\circ} \mathrm{C}$, on the same number of slides and the control section, showed no advantage. For $K$ pneumoniae we used a monoclonal antibody (catalogue No 5580-5208, Biogenesis, Poole, England), at a dilution of 1 in 6000 for $30 \mathrm{~min}-$ utes at room temperature.

All antibodies were tested by immunohistochemistry for cross reactivity, not only with the three bacterial species being investigated but also with the human intestinal sections injected with $P$ mirabilis. The mucus layer of control sections was identified by alcian blue/diastase and periodic acid Schiff staining, and primary antibodies only stained this layer when organisms were identified by Gram-Twort stain. Omission of the primary antibody was used as a negative control on tissue sections.

Slides were viewed by three investigators, two of whom were blinded as to the specific antibody used.

\section{Results}

Gram-Twort staining showed whole bacteria adjacent to the mucosa of normal and disease sections. Ulcer bases in either Crohn's or ulcerative colitis have very few whole bacteria on the luminal surface and none was identified within ulcer slough.

All positive control tissues stained positive with the appropriate antibody. There was no cross reactivity with any of the antibodies up to dilution of 1:2000, and staining of mucus only occurred when organisms where also seen by Gram-Twort. The antibody against $K$ pneumoniae detected all seven capsular subtypes investigated.

Positive specific staining of bacteria within the bowel lumen or held in the mucus layer was not taken as significant and did not differ between diseased tissues and controls.

In the Crohn's disease cases, positive staining for $E$ coli was found in ulcer slough of six, with a further two cases showing staining on or within surface enterocytes (35\% of cases). There were no cases positive for $L$ monocytogenes. One case stained positively for $K$ pneumonia in ulcer slough.

In the ulcerative colitis cases, five stained positive for $E$ coli antigen-three in ulcer slough, one within a crypt abscess, and one on the surface epithelium. One case was positive for $L$ monocytogenes on the edge of an ulcer. One case was positive for $K$ pneumonia within slough at the base of an ulcer.

Only one case stained positively for more than one bacterial antigen-an ulcerative colitis case showing marked acute inflammation with extensive ulceration and pseudopolyp formation, which stained for both $E$ coli and $K$ pneumoniae in different areas of ulceration.

Normal tissues from cancer resection specimens were all negative for $E$ coli, Kpneumoniae, and $L$ monocytogenes antigens.

Areas of positive staining for all bacterial antigens were generally those with acute inflammation in close proximity to abundant polymorphonuclear cells. In Crohn's disease there was no positive staining for any of the antigens in any of a total of 77 granulomas reviewed, nor of any giant cells or germinal centres.

Examples of the staining seen for $E$ coli are shown in fig 1 , that of $L$ monocytogenes in fig 2 , and for K pneumoniae in fig 3.

\section{Discussion}

In this study we failed to find evidence of $L$ monocytogenes in Crohn's disease tissues. E coli antigens were found in a minority of cases of Crohn's disease within ulcer slough or in a superficial setting, and not in deeper tissues including granulomas or giant cells. K pneumoniae antigens were also found to be present superficially in two cases-one case each of ulcerative colitis and Crohn's disease.

Despite the recognition of the contributory role of genetic factors in the development inflammatory bowel disease, ${ }^{14}$ there remains a substantial environmental contribution to the pathogenesis of these chronic gastrointestinal disorders. ${ }^{15}$ The concept that inflammatory 

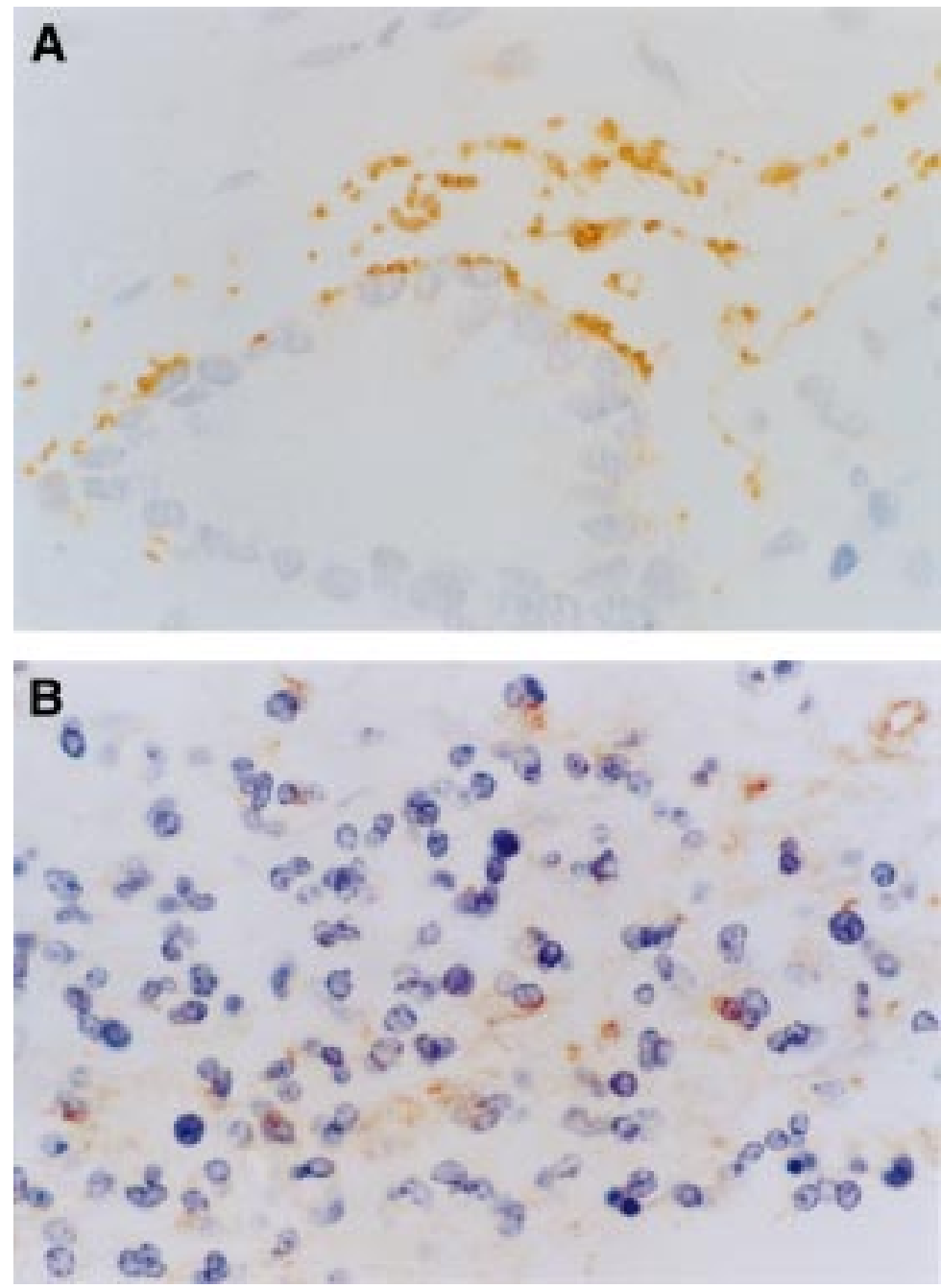

Figure 3 Staining for Klebsiella pneumoniae in $(A)$ positive control section of normal human small bowel injected submucosally with K pneumoniae subtype 50. (B) A case of ulcerative colitis showing positive staining within the slough of an ulcer.

bowel disease might be caused by specific infectious agents is still popular. $E$ coli attracted particular early interest because of the presence of antibodies which cross react with a human colon derived antigen and the Kunin antigen of $E$ coli and other enterobacteria, in patients with both Crohn's disease and ulcerative colitis. ${ }^{16}$ In addition, $E$ col $i$ with increased adhesive properties have been isolated from ulcerative colitis patients, ${ }^{17} 18$ suggesting a direct role in the pathogenesis. Antibodies to K pneumoniae have been found by several groups, including ours, to be present in patients with both inflammatory bowel disease and ankylosing spondylitis. $^{8-1019}$ It has been proposed that molecular mimicry between klebsiella antigens and the HLA-B27 molecule could be a pathogenic mechanism. Patients with ankylosing spondylitis appear to have occult bowel inflammation in up to $67 \%$ of $\operatorname{cases}^{20}$ and undiagnosed Crohn's disease in around $25 \% .^{21}{ }^{22} \mathrm{We}$ therefore investigated for persistence of $K$ pneumoniae antigens in inflammatory bowel disease tissues but were unable to find any evidence of this, even in biopsies of a patient with ulcerative colitis and ankylosing spondylitis.
Our study is at variance with the work of Liu et $a l,{ }^{12}$ both in the number of cases staining positive for $E$ coli or L monocytogenes and in the staining pattern. Faecal colonisation with $L$ monocytogenes species is not universal ${ }^{23}$ and it is possible that the carriage rate in the areas of France, where the majority of their cases originated, is different from that of our population. Liu and his colleagues' interpretation of the pattern of staining seen in their study was that it indicated the presence of intracellular bacterial antigens. We lacked suitable control tissue to prove whether these antibodies - which in the case of $E$ coli and $L$ monocytogenes were identical to those used by Liu et al-could detect intracellular organisms; however, the staining pattern seen in fig $1 \mathrm{C}$ suggests that this was the case. Highly sensitive molecular biological techniques have now been used to investigate differences in bacterial load and species range in the colonic mucosal biopsies of control and inflammatory bowel disease patients. ${ }^{24}$ Khilkin et al, using polymerase chain reaction, found an increased bacterial load in the inflamed mucosa of patients with a diagnosis of idiopathic inflammatory bowel disease as well as those with inflammation of other causes. $^{24}$ There were no differences in the species composition between the inflammatory bowel disease and non-inflammatory bowel disease groups. The exact microscopic localisation of any bacteria involved will give more clues as to their relevance to the pathophysiology of the disease. The distribution of staining of bacterial antigens in our study, being predominantly in ulcer slough and occasionally in the epithelium, is compatible with what we understand of the altered mucosal mucus defences in inflammatory bowel disease. ${ }^{25}$ Duchmann and colleagues have found that cellular and humoral mucosal immunity towards the resident faecal flora is increased in active inflammatory bowel disease ${ }^{26}$ but does not appear to be defective, and so one would expect bacteria penetrating the mucosal defences to be dealt with effectively. We propose that these bacteria therefore only play a secondary role in the pathogenesis of the idiopathic inflammatory bowel diseases.

In conclusion we have only detected $E$ coli, $L$ monocytogenes, and $K$ pneumoniae in superficial areas of inflammatory bowel disease resection specimens and not in deeper tissues. These findings do not support the suggestion that these bacteria play a primary aetiological role in inflammatory bowel disease.

We thank Dr Alan Philips of the Department of Paediatric Gastroenterology, Royal Free Hospital, for the donation of the $E$ coli O114 positive biopsies. RSW is in receipt of the 1996 Royal College of Physicians BUPA Fellowship.

1 Rutgeerts P, Goboes K, Peeters M, et al. Effect of faecal stream diversion on recurrence of Crohn's disease in the neoterminal ileum. Lancet 1991;338:771-4.

2 Rutgeerts P, Heile M, Geboes K, et al. Controlled trial of metronidazole treatment for prevention of Crohn's recurrence after ileal resection. Gastroenterology 1995;108:161721.

3 Gardiner KR, Halliday MI, Barclay GR, et al. Significance of systemic endotoxaemia in inflammatory bowel disease. Gut 36:897-901.

4 Sadlack B, Merz H, Schorle H, et al. Ulcerative colitis-like disease in mice with a disrupted interleukin-2 gene. Cell 1993;75:253-61. 
5 Kuhn R, Lohler J, Rennick D, et al. Interleukin-10 deficient mice develop chronic enterocolitis. Cell 1993;75:263-74.

6 Wensinck F, van de Merwe JP. Serum agglutinins to eubacterium and peptostreptococcus species in Crohn's and other diseases. $\mathscr{f}$ Hygiene 1981;87:13-24.

7 Auer IO, Rhoder A, Wensinck F, et al. Selected bacterial antibodies in Crohn's disease and ulcerative colitis. Scand $\mathcal{F}$ Gastroenterol 1983;18:217-23.

8 Ibbotson JP, Pease PE, Allan RE. Serological studies in Crohn's disease. Eur f Clin Microbiol 1987;6:286-90.

9 Cooper R, Fraser SM, Sturrock RD, et al. Raised titres of anti-klebsiella IgA in ankylosing spondylitis, rheumatoid arthritis and inflammatory bowel disease. BMF 1988;296: 1432-4.

10 Tiwana H, Wilson C, Walmsley RS, et al. Antibody responses to gut bacteria in ankylosing spondylitis rheumatoid arthritis, Crohn's disease and ulcerative colitis. Rheumatol Int 1997; 17:11-16.

11 Klasen IS, Melief MJ, van Halerteren AGS, et al. The presence of peptidoglycan-polysaccharide complexes in the bowel wall and the cellular responses to these complexes in bowel wall and the cellular responses to these complexes in Cro

12 Liu Y, Van Kruningen J, West AB, et al. Immunocytochemical evidence of listeria, Escherichia coli and streptococcus antigens in Crohn's disease. Gastroenterology 1995;108 1396-404.

13 Ollett WS. A method for staining both Gram positive and Gram negative bacteria in sections. F Pathol Bacteriol 1947; 59:357.

14 Satsanghi J, Welsh KI, Bunce M, et al. Contribution of genes of the major histocompatibility complex to susceptibility and disease phenotype in inflammatory bowel disease. Lancet 1996;347:1212-17.

15 Gent A, Hellier MD, Grace RH, et al. Inflammatory bowel disease and domestic hygiene in infancy. Lancet 1994;343: $766-7$.
16 Lagercrantz R, Hammarstrom S, Perlmann $\mathrm{P}$, et al. Immunological studies in ulcerative colitis IV: origin of autoantibodies. F Exp Med 1968;128:1339-52.

17 Dickinson RJ, Varian SA, Axon AT, et al. Increased incidence of faecal isoforms with in vitro adhesive and invasive properties in patients with ulcerative colitis. Gut 1980;21:787-92.

18 Burke DA, Axon AT. Ulcerative colitis and Escherichia coli with adhesive properties. 7 Clin Pathol 1987;40:782-6.

19 O'Mahony S, Anderson N, Nuki G, et al. Systemic and mucosal antibodies to klebsiella in patients with ankylosing spondylitis and Crohn's disease. Ann Rheum Dis 1992;51: 1296-300.

20 Mielants H, Veys EM, Cuvelier C, et al. Ileocolonoscopic findings in seronegative spondylarthropathies. $\mathrm{Br} \mathcal{F}$ Rheumatol 1988;27(suppl II):95-105.

21 de Vos $M$, Mielants $H$, Cuvelier $C$, et al. Long-term evolution of gut inflammation in patients with spondyloarthropathy. Gastroenterology 1996;110:1696-703.

22 Leirisalo-Repo M, Turunen U, Stenman S, et al. High frequency of silent inflammatory bowel disease in spondyloarthropathy. Arthritis Rheum 1994;37:23-31.

23 Muller HE. Listeria isolations from faeces of patients with diarrhoea and from healthy food handlers. Infection 1990;18:97-100.

24 Khilkin M, Swidinski A, Schreiber S, et al. Bacterial invasion of the mucosa is an essential component of inflammation in inflammatory bowel disease [abstr]. Gastroenterology 1997; 112: A1012.

25 Rhodes JM. Mucins in inflammatory bowel disease. $Q \mathcal{F} M e d$ 1997;90:79-82

26 Duchmann R, Kaiser I, Hermann E, et al. Tolerance exists towards resident intestinal flora but is broken in active inflammatory bowel disease. Clin Exp Immunol 1995;102: $448-55$. 